\title{
Fatores associados à variação de hemoglobina em mulheres submetidas à hemodiálise
}

\author{
Factors associated with hemoglobin variation in women submitted to hemodialysis \\ Factores asociados con la variación de la hemoglobina en mujeres sometidas a hemodiálisis
}

Recebido: 22/05/2021 | Revisado: 29/05/2021 | Aceito: 01/06/2021 | Publicado: 16/06/2021

\author{
Danielle Alice Vieira da Silva \\ ORCID: https://orcid.org/0000-0002-5180-2607 \\ Universidade Federal de Alagoas, Brasil \\ E-mail: danielle.silva@fanut.ufal.br \\ Lidyane Cerqueira de Araujo Silva \\ ORCID: https://orcid.org/0000-0001-7956-6688 \\ Centro Universitário Tiradentes, Brasil \\ E-mail: lidyanea10@gmail.com \\ Ícaro Carlos Oliveira de Andrade \\ ORCID: https://orcid.org/0000-0002-0171-553X \\ Centro Universitário Tiradentes, Brasil \\ E-mail: icaro.carlos@souunit.com.br \\ Ayná Santos de Lima \\ ORCID: https://orcid.org/0000-0001-6364-2922 \\ Centro Universitário Tiradentes, Brasil \\ E-mail: aynalima@hotmail.com \\ João Paulo da Silva Lima \\ ORCID: https://orcid.org/0000-0001-5864-0295 \\ Centro Universitário Tiradentes, Brasil \\ E-mail: nutrijohn@hotmail.com \\ Ana Debora Santos de Oliveira \\ ORCID: https://orcid.org/0000-0001-9857-1327 \\ Universidade Federal de Alagoas, Brasil \\ E-mail: anadebora.db@gmail.com
}

\section{Resumo}

Avaliar os fatores associados ao declínio da hemoglobina em pacientes com DRC. Trata-se de um estudo transversal, conduzido com mulheres submetidas à hemodiálise, excetuando-se as portadoras de doenças hemolíticas. A coleta de dados bioquímicos e antropométricos foram realizadas a partir de prontuário eletrônico das pacientes inseridas na pesquisa. Cerca de $80 \%$ das mulheres eram adultas e o agravo crônico de maior prevalência foi a Hipertensão Arterial Sistêmica (75,65\%). Foi possível constatar que todas as categorias analisadas apresentaram média de hemoglobina com valor inferior a $12 \mathrm{~g} / \mathrm{dL}$ indicando a anemia como um problema em vigência no público estudado. Os fatores que mostraram associação estatisticamente significativa com os menores níveis de hemoglobina foram: Hipertensão, Diabetes mellitus e o menor tempo em hemodiálise. A presença de outros agravos no portador de Doença Renal Crônica, como diabetes, hipertensão e menor tempo de hemodiálise, contribuem para maior comprometimento dos níveis de hemoglobina.

Palavras-chave: Doença renal crônica; Anemia; Hemodiálise.

\begin{abstract}
To evaluate the factors associated with the decline in hemoglobin in patients with CKD. This is a cross-sectional study, conducted with women undergoing hemodialysis, except for those with hemolytic diseases. The collection of biochemical and anthropometric data was carried out from the electronic medical records of the patients included in the research. $80 \%$ of the women were adults and the most prevalent chronic condition was Systemic Arterial Hypertension (75.65\%). It was possible to verify that all the categories analyzed presented an average hemoglobin value below $12 \mathrm{~g} / \mathrm{dL}$, indicating anemia as a problem in effect in the studied public. The factors that showed a statistically significant association with the lowest hemoglobin levels were: Hypertension, Diabetes mellitus and the shortest time on hemodialysis. The presence of other conditions in patients with CKD, such as diabetes, hypertension and shorter hemodialysis time, contribute to greater impairment of hemoglobin levels.
\end{abstract}

Keywords: Chronic kidney disease; Anemia; Hemodialysis.

\section{Resumen}

Evaluar los factores asociados al descenso de la hemoglobina en pacientes con ERC. Se trata de un estudio transversal, realizado con mujeres en hemodiálisis, excepto aquellas con enfermedades hemolíticas. La recolección de datos bioquímicos y antropométricos se realizó a partir de la historia clínica electrónica de los pacientes incluidos en la investigación. El 80\% de las mujeres eran adultas y la condición crónica más prevalente fue la hipertensión arterial sistémica (75,65\%). Se pudo comprobar 
que todas las categorías analizadas presentaron un valor promedio de hemoglobina por debajo de $12 \mathrm{~g} / \mathrm{dL}$, lo que indica que la anemia es un problema en efecto en el público estudiado. Los factores que mostraron asociación estadísticamente significativa con los niveles más bajos de hemoglobina fueron: Hipertensión, Diabetes mellitus y el menor tiempo en hemodiálisis. La presencia de otras afecciones en pacientes con ERC, como diabetes, hipertensión y menor tiempo de hemodiálisis, contribuyen a un mayor deterioro de los niveles de hemoglobina.

Palabras clave: Enfermedad renal crónica, Anemia, Hemodiálisis.

\section{Introdução}

A Doença Renal Crônica (DRC) se desenvolve em detrimento da incapacidade renal na realização de suas funções de forma satisfatória. À medida em que ocorre a progressão do agravo, faz-se necessário o uso de medicamentos, intervenções nutricionais e, em casos mais graves, tratamentos dialíticos (Prestes et al., 2011). Atualmente, a DRC é considerada um problema de saúde pública mundial, no Brasil cerca de 1,4 milhões de indivíduos possuem algum grau de perda da função renal (Ribeiro, Jorge \& Queiroz, 2020), é uma doença marcada pela progressão da lesão, e consequente redução da taxa de filtração glomerular (TFG), a hemodiálise é o tratamento terapêutico mais utilizado e tem como objetivo manter as funções anteriormente desempenhadas pelos rins (Rosa \& Loures, 2013).

Esse agravo é marcado pela perda lenta e progressiva da função renal e consequente redução da taxa de filtração glomerular (TFG), sendo a hemodiálise o tratamento de terapia renal substitutiva mais utilizado, sendo também necessário o uso de medicamentos e intervenções nutricionais adequadas (Rosa \& Loures, 2013, Prestes et al., 2011). Atualmente, a DRC é considerada um problema de saúde pública mundial. No Brasil, cerca de 1,4 milhões de indivíduos possuem algum grau de perda de função renal (Ribeiro, Jorge \& Queiroz, 2020).

A literatura descreve diversas alterações que podem surgir na vigência da DRC e da hemodiálise, sendo a anemia uma das mais comuns. Dentre as causas do surgimento da anemia em portadores de DRC, tem-se a diminuição no número de células peritubulares renais e da síntese de eritropoetina (EPO), sendo está uma glicoproteína que em casos de hipóxia do tecido renal, estimula a eritropoiese enquanto quantidades suficientes de eritrócitos sejam produzidas para transporte de oxigênio (Penteado, Lima \& Milagres, 2017, Santos et al., 2020). Destaca-se também que a desordem costumeiramente começa a se manifestar no momento em que a TFG apresenta valores abaixo de $60 \mathrm{mg} / \mathrm{m}$, havendo uma relação direta entre redução da TFG e a piora do quadro da anemia (Shaikh \& Aeddula, 2020).

Como agravante, um estudo aponta que pacientes sujeitos cronicamente à hemodiálise são especialmente vulneráveis à deficiência de ferro, devido às perdas de sangue durante a sessão, sangramento e absorção gastrointestinal comprometida deste metal. Sua ocorrência associada à DRC é um tópico de constante preocupação por parte dos nefrologistas, uma vez que aumenta significativamente a morbidade e mortalidade destes pacientes (Basic-Jukic et al., 2006).

Assim, o presente estudo teve como objetivo avaliar os fatores associados ao declínio da hemoglobina em pacientes com DRC, submetidos a hemodiálise em um centro de referência em uma capital nordestina.

\section{Metodologia}

\subsection{Desenho do estudo e aspectos éticos}

Trata-se de um trabalho de delineamento transversal, quantitativo, segundo as recomendações estabelecidas por Estrela (2018), com dados secundários, advindos de um projeto previamente aprovado sob parecer de número 3.356.492, realizado em um centro de referência em hemodiálise do estado de Alagoas. 


\subsection{Critérios de inclusão e exclusão}

Foi adotado como critério de inclusão: ser do sexo feminino, apresentar idade mínima de 18 anos, ter diagnóstico de DRC, o qual foi confirmado com o cálculo da TFG pela fórmula de Cockcroft-Gault, e estar em tratamento hemodialítico há pelo menos 30 dias. Ademais, foram excluídas possíveis participantes que apresentassem alguma doença hemolítica.

\subsection{Coleta dos dados}

Todas as pacientes foram conscientizadas dos objetivos da pesquisa, sendo incluídas na amostra apenas as que assinaram o Termo de Consentimento Livre e Esclarecido (TCLE). Por meio de pesquisadores treinados, e com uso de um formulário próprio, foram coletados dados de caracterização socioeconômica e demográfica.

Para coleta dos dados bioquímicos e antropométricos foram consultados os prontuários eletrônicos dos pacientes inseridos na pesquisa. O prontuário digital das pacientes apresentava-se sob a forma de um software, a saber o Smart Health v01.11, onde constava o registro de peso seco $(\mathrm{kg})$ no pós-diálise e altura $(\mathrm{m})$. A partir desses dados foi calculado o índice de massa corporal $(\mathrm{IMC})\left(\mathrm{kg} / \mathrm{m}^{2}\right)$. Também foi registrada a data de início do tratamento dialítico (dia/mês/ano) e concentração de hemoglobina sérica (g/dL). Foram coletados os dados mais recentes de hemoglobina de cada paciente, não ultrapassando 60 dias, já que periodicamente estes passam por avaliação laboratorial. Os dados antropométricos coletados do prontuário foram mensurados pela nutricionista responsável pelo setor.

\subsection{Tabulação e análise dos dados}

Os dados de IMC foram categorizados em: baixo peso $\left(<18,5 \mathrm{~kg} / \mathrm{m}^{2}\right)$ e eutrofia $\left(\geq 18,5 \mathrm{~kg} / \mathrm{m}^{2}\right)$ (OMS, 2000). Já quanto a concentração sérica de hemoglobina, classificou-se da seguinte forma: $\leq 13,9 \mathrm{~g} / \mathrm{dL}$ (nível reduzido), 14 - 17,5 g/dL (normal) e $\geq 17,6 \mathrm{~g} / \mathrm{dL}$ (aumentado). O diagnóstico de anemia foi adotado para aquelas que apresentaram níveis reduzido de hemoglobina, portanto, $\leq 13,9 \mathrm{~g} / \mathrm{dL}$.

Os resultados foram tabulados no Excel® versão 2003 e as análises estatísticas realizadas com o software SPSS (Statistical Package for the Social Sciences, versão 22.0, SPSS Inc, Chicago, Illinois, EUA). Primeiramente, realizou-se o teste de Shapiro-Wilk para verificação da normalidade e as variáveis foram expressas em valor absoluto, percentual, média e desvio padrão (DP). Para comparação das médias, foi utilizado o teste T para as variáveis com distribuição normal e o teste de MannWhitney para as não paramétricas. Foi admitido um valor de 5\% para estabelecer a relação de significância estatística.

\section{Resultados}

Dos 300 pacientes assistidos no hospital no momento da pesquisa, 85 atendiam aos critérios de inclusão, entretanto 7 desses pacientes se recusaram a participar da pesquisa, o que correspondeu a uma perda de 8,23\%. Por fim, 78 pacientes completaram o estudo em questão, o que correspondeu a $91.77 \%$ do número de pacientes do sexo feminino, elegíveis que deram início a pesquisa.

O apanhado dos dados coletados a partir da aplicação dos questionários, os quais contemplaram dados socioeconômicos, demográficos, pessoais e de estilo de vida está exposto na Tabela 1. Foi possível verificar que quase $80 \%$ são adultos e que o agravo crônico de maior prevalência foi a Hipertensão Arterial Sistêmica (HAS), seguido de diabetes mellitus e tempo em hemodiálise. Quanto à renda familiar, mais da metade da amostra recebia até 1 salário mínimo e mais de 3/4 das entrevistadas estavam eutróficas. 
Research, Society and Development, v. 10, n. 7, e13710716387, 2021

(CC BY 4.0) | ISSN 2525-3409 | DOI: http://dx.doi.org/10.33448/rsd-v10i7.16387

Tabela 1 - Caracterização socioeconômica, demográfica e de saúde de pacientes em hemodiálise, Maceió, AL, 2020.

\begin{tabular}{|c|c|c|}
\hline \multirow[t]{2}{*}{ Variáveis } & \multicolumn{2}{|c|}{ População total } \\
\hline & $\mathrm{n}=\mathbf{7 8}$ & Percentual (\%) \\
\hline \multicolumn{3}{|l|}{ Idade } \\
\hline Adulto (18-59 anos) & 61 & 78,21 \\
\hline Idoso (> 60 anos) & 17 & 21,79 \\
\hline \multicolumn{3}{|l|}{ Hipertensão Arterial Sistólica (HAS) } \\
\hline Sim & 59 & 75,65 \\
\hline Não & 19 & 24,35 \\
\hline \multicolumn{3}{|l|}{ Diabetes Mellitus (DM) } \\
\hline Sim & 33 & 42,31 \\
\hline Não & 45 & 57,69 \\
\hline \multicolumn{3}{|l|}{ Escolaridade } \\
\hline Analfabeto/Fundamental & 51 & 65,39 \\
\hline Médio/Superior & 27 & 34,61 \\
\hline \multicolumn{3}{|l|}{ Moradia } \\
\hline Própria/ cedida & 60 & 76,93 \\
\hline Alugada & 18 & 23,07 \\
\hline \multicolumn{3}{|l|}{ Localização moradia } \\
\hline Zona urbana & 67 & 85,89 \\
\hline Zona Rural/Zona Indígena & 11 & 14,11 \\
\hline \multicolumn{3}{|l|}{ Renda Familiar } \\
\hline Até 1 salário mínimo & 45 & 57,69 \\
\hline Acima de 1 salário Mínimo & 33 & 42,31 \\
\hline \multicolumn{3}{|l|}{ Tempo em Hemodiálise } \\
\hline Até 2 anos & 42 & 53,85 \\
\hline$>2$ anos & 36 & 46,15 \\
\hline \multicolumn{3}{|l|}{ Prática de atividade física } \\
\hline Sim & 33 & 42,31 \\
\hline Não & 45 & 57,69 \\
\hline \multicolumn{3}{|l|}{ Índice de Massa Corpórea (IMC) } \\
\hline Baixo peso $\left(<18,5 \mathrm{~kg} / \mathrm{m}^{2}\right)$ & 12 & 15,39 \\
\hline Eutrofia $\left(>18,5 \mathrm{~kg} / \mathrm{m}^{2}\right)$ & 66 & 84,61 \\
\hline \multicolumn{3}{|l|}{ Tabagismo } \\
\hline Sim & 35 & 44,88 \\
\hline Não & 43 & 55,12 \\
\hline \multicolumn{3}{|l|}{ Etilismo } \\
\hline Ex- etilista & 36 & 46,16 \\
\hline Não etilista & 42 & 53,84 \\
\hline
\end{tabular}


Através das variáveis apresentadas, foi possível constatar que todas as categorias aqui analisadas apresentaram média de hemoglobina com valor inferior a $12 \mathrm{~g} / \mathrm{dL}$, indicando a anemia como um problema em vigência no público estudado (Tabela 2).

Verificou-se que, a HAS, o DM e o tempo em hemodiálise de até 2 anos das participantes do estudo apresentaram pvalor inferior a 0,05 , indicando que esses fatores apresentaram relação estatisticamente significativa com a variável aqui estudada, a saber, menor concentração de hemoglobina sérica (Tabela 2).

Tabela 2 - Fatores associados às médias de hemoglobina de pacientes em hemodiálise, Maceió, AL, 2020.

\begin{tabular}{|c|c|c|c|c|c|}
\hline Variáveis & Absoluto & $\begin{array}{c}\text { Percentual } \\
\text { (\%) }\end{array}$ & $\begin{array}{c}\text { Média } \\
\text { Hemoglobina }\end{array}$ & DP & p -valor \\
\hline$\overline{\text { Adulto (18-59 anos) }}$ & 61 & 78,21 & 10,15 & 2,34 & 0.627 \\
\hline Idoso $(>60$ anos $)$ & 17 & 21,79 & 10,46 & 2,16 & \\
\hline Hipertenso & 59 & 75,65 & 9,89 & 2,12 & 0,025 \\
\hline Não- hipertenso & 19 & 24,35 & 11,24 & 2,56 & \\
\hline Diabético & 33 & 42,31 & 9,59 & 2,22 & 0,038 \\
\hline Não-diabético & 45 & 57,69 & 10,68 & 2,25 & \\
\hline Analfabeto/ & 51 & 65,39 & 10,42 & 2,39 & 0,302 \\
\hline \multicolumn{6}{|l|}{ Fundamental } \\
\hline Médio/Superior & 27 & 34,61 & 9,85 & 2,08 & \\
\hline Moradia Própria/ cedida & 60 & 76,93 & 10,25 & 2,24 & 0,833 \\
\hline Alugada & 18 & 23,07 & 10,12 & 2,50 & \\
\hline Zona urbana & 67 & 85,89 & 10,22 & 2,26 & 0,995 \\
\hline Zona rural/Zona indígena & 11 & 14,11 & 10,22 & 2,56 & \\
\hline Até 1 salário mínimo & 45 & 57,69 & 10,34 & 2,20 & 0,582 \\
\hline Acima de 1 salário & 33 & 42,31 & 10 & 2,44 & \\
\hline \multicolumn{6}{|l|}{ mínimo } \\
\hline Tempo hemodiálise $\leq 2$ anos & 42 & 53,85 & 9,50 & 2,11 & 0,002 \\
\hline$>2$ anos & 36 & 46,15 & 11,05 & 2,24 & \\
\hline Praticante de atividade física & 33 & 42,31 & 10,28 & 2,19 & 0,840 \\
\hline Não praticante & 45 & 57,69 & 10,17 & 2,38 & \\
\hline IMC (baixo peso) & 12 & 15,39 & 10,1 & 2,07 & 0,842 \\
\hline IMC (eutrofia) & 66 & 84,61 & 10,24 & 2,34 & \\
\hline Tabagista & 35 & 44,88 & 10,36 & 2,20 & 0,631 \\
\hline Não- tabagista & 43 & 55,12 & 10,10 & 2,38 & \\
\hline Ex- Etilista & 36 & 46,16 & 10,05 & 2,30 & 0,548 \\
\hline Não Etilista & 42 & 53,84 & 10,36 & 2,30 & \\
\hline
\end{tabular}

Vigência do salário mínimo atual: R \$1.045.

Fonte: Dados da pesquisa (2020). 


\section{Discussão}

Segundo a Organização Mundial da Saúde (OMS), a anemia pode ser definida como a condição na qual a concentração sanguínea de hemoglobina se encontra abaixo dos valores esperados (inferior a - 2DP), tornando-se insuficiente para atender as necessidades fisiológicas exigidas de acordo com idade, sexo, gestação e altitude (OMS, 2015). Comumente, estes valores referem-se a concentrações sanguíneas abaixo de 13g/dL, para homens, e 12g/dL, para mulheres (Shaikh \& Aeddula, 2020).

Embora tratável, percebe-se que a incidência da anemia nos pacientes com DRC aumenta à medida que se avançam os estágios da doença (Hazin, 2020). O fato de a amostra aqui estudada estar acometida majoritariamente por um grave comprometimento da TFG pode explicar as baixas médias de hemoglobina em todas as categorias estudadas.

Stauffer e Fan (2014), em estudo avaliando a prevalência de anemia em pacientes com DRC nos Estados Unidos constataram que mais de $50 \%$ dos pacientes com diagnóstico de DRC nos estágios 4 e 5 também apresentaram anemia, onde os estágios eram estabelecidos com base nos valores calculados para TFG e evidência de dano renal, de acordo com as diretrizes da National Kidney Foundation (2012). Em concordância com os achados citados, neste estudo, cuja amostra também foi composta por pacientes com DRC avançada, sobretudo nível 5, constatou-se que 59 das 78 pacientes (75,64\%), portadoras de DRC, também apresentavam algum grau de anemia.

Com relação aos fatores associados ao comprometimento nos níveis de hemoglobina, tem-se a HAS. Sua associação com a DRC apresenta uma relação circular entre si, onde ambas são causa e consequência da outra. A HAS pode ser encontrada em 80 - 85\% de todos os pacientes com DRC, com maior prevalência sendo vista nos estágios mais avançados da doença (Gargiulo, Suhail \& Lerma, 2015). No guideline: “Evidence-Based Guideline for the Management of High Blood Pressure in Adults Report From the Panel Members Appointed to the Eighth Joint National Committee" publicado no Journal of the American Medical Association (JAMA), vários estudos identificam a HAS mal controlada como um importante fator de risco para progressão da DRC para a doença renal de estágio final.

Os níveis normais de pressão são geralmente mantidos rigidamente por meio de um processo chamado "natriurese pressórica"' e inclui a participação dos rins para excreção do excesso de sódio. As alterações associadas à DRC resultam em menor número de néfrons e troca tubular renal prejudicada, resultando em declínio na excreção de sódio e fluidos em um ambiente hipertensivo com prejuízo na eficiência com que se amortece as elevações pressóricas por meio deste mecanismo (Gargiulo, Suhail \& Lerma, 2015).

Outro sistema envolvido na regulação dos níveis pressóricos e que pode sofrer interferência com a DRC é o sistema renina - angiotensina - aldosterona (RAA), o qual tem sua atividade aumentada neste caso. A excreção aumentada de renina a partir dos rins na DRC, possivelmente resultado de isquemia renal, é tida como a causa mais provável para a atividade anormal. Vários estudos demonstram efetividade de fármacos com atividade bloqueadora do sistema RAA em reduzir os níveis de pressão sanguínea, melhorar quadro de proteinúria e reduzir a progressão da doença renal, desta forma, potencialmente contribuindo para minimização da progressão da anemia nestes pacientes, como menciona o artigo de revisão escrito por Viazzi, Leoncini \& Pontremoli (2013).

Quando há menores concentrações de hemoglobina ocorre o aumento do trabalho miocárdico para compensação da hipóxia tecidual; com o tempo, a hipertrofia ventricular esquerda se desenvolve; e os fenômenos associados a essa alteração incluem isquemia cardíaca, insuficiência cardíaca e infarto miocárdico. A hipertrofia ventricular esquerda está associada a concentrações de hemoglobina inferiores a 12g/dL (BREGMANN, 2009).

Quando não há compatibilidade entre os valores hematimétricos com a TFG, faz-se necessário investigar outras possíveis causas como sangramento crônico, deficiência de vitamina B12 e ferro, os efeitos medicamentosos utilizados durante a hemodiálise, sobretudo a diminuição na produção renal de EPO (Soares, 2015). 
Outra variável aqui relacionada com menores valores de hemoglobina foi a presença de DM. Em um estudo foi investigada a prevalência da anemia de acordo com o nível da função renal e o status de DM dos avaliados. Os indivíduos foram divididos em quatro categorias de acordo com os valores estimados para a TFG da seguinte forma: grupo 1 (TFG $\geq 90$ ), grupo 2 (TFG entre 60 - 89), grupo 3 (TFG entre $30-59)$, e grupo $4\left(\mathrm{TFG}<30 \mathrm{~mL} / \mathrm{min} / 1.73 \mathrm{~m}^{2}\right)$. Em participantes com o diagnóstico de DM a prevalência de anemia entre os grupos foi de $8.7 \%, 7.5 \%, 22.2 \%$, e 52,4\%, respectivamente, comparada a $6.9 \%, 5.0 \%$, $7.9 \%$, e 50,0\% para indivíduos sem o diagnóstico de DM. O estudo então concluiu que a DM se associou de forma independente e pode estar relacionada à expressão prematura de anemia em pessoas com redução moderada da função renal (El-Achkar et al., 2005).

Como já mencionado, a causa mais comum de anemia na DRC é a deficiência de EPO, sendo está deficiência mais pronunciada em indivíduos diabéticos, como ilustra o estudo de Symeonidis et al., (2006). Neste estudo, avaliaram-se 694 pacientes anêmicos e constatou-se que, em relação a gravidade da anemia, os pacientes diabéticos apresentavam menor concentração sérica de EPO em relação aos não diabéticos, com exceção casos de anemia megaloblástica e anemia de doenças mieloproliferativas.

Além da glicosilação enzimática (covalente), a molécula de EPO também pode ser glicada de forma não covalente, sendo a taxa desse processo fortemente dependente dos níveis séricos de glicose. Com isso, a glicação não enzimática pode ser capaz de alterar as propriedades físico-químicas da EPO, em particular, sua taxa de catabolismo. Dessa forma, é possível que em indivíduos diabéticos a EPO tenha a sua produção normal, por outro lado, seu catabolismo acelerado, como resultado de uma glicação aumentada (Maiese, 2015).

Sabendo que a toxicidade urêmica e a inflamação são capazes de exacerbar a anemia da DRC, afetando a eritropoiese da médula óssea e a sobrevivência dos eritrócitos, pode-se ter na hemodiálise uma ferramenta terapêutica importante graças a sua capacidade de afetar diretamente a eritropoiese da medula óssea, tanto por remover substâncias capazes de inibir a eritropoiese, quanto por aumentar a disponibilidade do ferro (Krause et al., 2008).

No presente estudo também foi evidenciado a associação entre o tempo (em meses) em hemodiálise e melhores valores de concentração plasmática de Hemoglobina, achado reforçado pelo estudo de Bowry e Gatti (2011). Neste último, após revisão de evidências que sugeriam que a prática da hemodiálise seria capaz de modular processos chaves envolvidos com a patogênese da anemia e com a sua terapia, concluiu-se que a sua prática impacta a anemia não só eliminando os inibidores urêmicos putativos que suprimem a eritropoiese, mas também reduzindo a destruição dos eritrócitos e aumentando a disponibilidade do ferro, assim como também por mecanismos que modulam a inflamação subjacente e a disfunção endotelial, primordiais para a gênese da DRC e da própria anemia. Um tratamento minucioso da HAS, controle da glicemia nos diabéticos, correção da anemia e prevenção da desnutrição parecem ser alguns dos fatores importantes na obtenção de uma intervenção clínica eficaz nos pacientes com DRC (Santos et al., 2020).

\section{Conclusão}

Neste estudo, HAS, DM e menor tempo em hemodiálise foram identificados como fatores associados a menor concentração sérica de hemoglobina, sugerindo que essas condições corroboram para o quadro de anemia, agravo comum em pacientes com DRC em tratamento hemodialítico.

Reforça-se, a importância da identificação de fatores que predisponham ao comprometimento da hemoglobina, uma vez que o quadro de anemia em pacientes com DRC contribuem para desfechos negativos. Assim, resultados de pesquisas como a aqui apresentada, contribuem no planejamento de intervenções/tratamento e prevenção de complicações. Salienta-se ainda que mais estudos devem ser fomentados, incluindo outros marcadores envolvidos na anemia que possam influenciar nos níveis de hemoglobina. 


\section{Referências}

Bragman R. (2009). Anemia na Doença Renal Crônica. Braz. J. Nephrol., 31 (1 suppl 1), 36-41. https://www.bjnephrology.org/en/article/anemia-na-doencarenal-cronica/

Bowry, S. K., \& Gatti, E. (2011). Impact of Hemodialysis Therapy on Anemia of Chronic Kidney Disease: The Potential Mechanisms. Blood Purification, 32(3), 210-219. doi:10.1159/000329573

El-Achkar, T. M., Ohmit, S. E., Mccullough, P. A., Crook, E. D., Brown, W. W., Grimm, R., Flack, J. M. (2005). Higher prevalence of anemia with diabetes mellitus in moderate kidney insufficiency: The Kidney Early Evaluation Program. Kidney International, 67(4), 1483-1488. doi:10.1111/j.15231755.2005.00226.x

Gargiulo, R., Suhail, F., \& Lerma, E. V. (2015). Hypertension and chronic kidney disease. Disease-a-Month, 61(9), 387-395. doi:10.1016/j.disamonth.2015.07.003

Hazin, M. A. (2020). Anemia in chronic kidney disease. Revista Da Associação Médica Brasileira, 66(Suppl 1). doi:10.1590/1806-9282.66.s1.55

Kruse, A., Uehlinger, D. E., Gotch, F., Kotanko, P., \& Levin, N. W. (2008). Red Blood Cell Lifespan, Erythropoiesis and Hemoglobin Control. Hemodialysis From Basic Research to Clinical Trials Contributions to Nephrology, 247-254. doi:10.1159/000130698

Maiese, K. (2015). Erythropoietin and diabetes mellitus. World Journal of Diabetes, 6(14), 1259. doi:10.4239/wjd.v6.i14.1259

Penteado, B. A.., Lima, W. F., Milagres, C. S. (2017). Etiologia de anemia em pacientes renais crônicos em tratamento hemodialítico. Rev. Cien. FHO, v. 5, n. 1, p. 18-23.

Prestes, F. C., et al (2011). Percepção dos trabalhadores de enfermagem sobre a dinâmica do trabalho e os pacientes em um serviço de hemodiálise. Text. Cont. Enferm., v. 20, n. 1, p. 25-32.

Ribeiro, W. A., Jorge B. O., Queiroz, R. S. (2020). Repercussões da hemodiálise no paciente com doença renal crônica. Revista Pró-UniverSUS, v. 11, n. 1, p. $88-97$.

Rosa, K. R.., Loures, M. C. (2013). Qualidade de vida de idosos em hemodiálise. EVS, v. 40, n. 4, p. 419-446.

Santos, N. C., et al. (2020). O papel do Enfermeiro na anemia associada à doença renal crônica. Research Society and Development, v. 9, n. 8, p. 1-16.

Shaikh, H.., Aeddula, N. R. (2020). Anemia Of Chronic Renal Disease. https://www.ncbi.nlm.nih.gov/books/NBK539871/

Sociedade Brasileira de Nefrologia (2017). Tratamento Hemodialítico. https://sbn.org.br

Stauffer, M. E., \& Fan, T. (2014). Prevalence of Anemia in Chronic Kidney Disease in the United States. PLoS ONE, 9(1). doi:10.1371/journal.pone.0084943

Symeonidis, A., Kouraklis-Symeonidis, A., Psiroyiannis, A., Leotsinidis, M., Kyriazopoulou, V., Vassilakos, P., Zoumbos, N. (2005). Inappropriately low erythropoietin response for the degree of anemia in patients with noninsulin-dependent diabetes mellitus. Annals of Hematology, 85(2), 79-85. doi: $10.1007 / \mathrm{s} 00277-005-1102-9$

Ulijaszek, S. J. (2003). Obesity: Preventing and Managing the Global Epidemic. Report of a WHO Consultation. WHO Technical Report Series 894 . Pp. 252.

Viazzi, F., Leoncini, G., \& Pontremoli, R. (2013). Antihypertensive Treatment and Renal Protection: The role of drugs inhibiting the renin-angiotensinaldosterone system. High Blood Pressure \& Cardiovascular Prevention, 20(4), 273-282. doi:10.1007/s40292-013-0027-y

Wish, J., Aronoff, G., Bacon, B., Brugnara, C., Eckardt, K. \& Ganz, T., Wood, J. (2018). Positive Iron Balance in Chronic Kidney Disease: How Much is Too Much and How to Tell? American Journal of Nephrology, 47(2), 72-83. doi:10.1159/000486968

World Health Organization (WHO) (2015). The global prevalence of anaemia in 2011. Geneva: World Health Organization. pag. 43.

World Health Organization (WHO). Obesity - Preventing and managing the global epidemic: Report of a WHO consultation. Geneva: World Health Organization, 2000, pag. 268. 